\title{
Interplay between multiple scattering and optical nonlinearity in liquid crystals
}

\author{
Alessandro Alberucci ${ }^{1,},{ }^{*}$, Chandroth P. Jisha ${ }^{2}$, Serena Bolis ${ }^{3,4}$, Jeroen Beeckman $^{4}$, and \\ STEFAN NOLTE ${ }^{1,5}$ \\ ${ }^{1}$ Institute of Applied Physics, Abbe Center of Photonics, Friedrich-Schiller-Universität Jena, Albert-Einstein-Str. 15, 07745 Jena, Germany \\ ${ }^{2}$ Centro de Física do Porto, Faculdade de Ciências, Universidade do Porto, Porto 4169-007, Portugal \\ ${ }^{3}$ Université libre de Bruxelles, OPERA-Photonics Group, 50 Avenue F.D.Roosevelt CP 194/5 1050 Bruxelles Belgium \\ ${ }^{4}$ Ghent University, ELIS Department, Technologiepark-Zwijnaarde 15, 9052 Gent, Belgium \\ ${ }^{5}$ Fraunhofer Institute of Applied Optics and Precision Engineering, Albert-Einstein-Straße 7, 07745 Jena, Germany \\ *Corresponding author: alessandro.alberucci@gmail.com
}

We discuss the role played by time-dependent scattering on light propagation in liquid crystals. In the linear regime, the effects of the molecular disorder accumulate in propagation, yielding a monotonic decrease in the beam spatial coherence. In the nonlinear case, despite the disorder-imposed Brownian-like motion to the self-guided waves, self-focusing increases the spatial coherence of the beam by inducing spatial localization. Eventually, a strong enhancement in the beam oscillations occurs when power is strong enough to induce self-steering, i.e., in the non-perturbative regime. (C) 2018 Optical Society of America

OCIS codes: (160.3710) Liquid Crystals; (190.6135) Spatial Solitons; (290.4210) Multiple Scattering; (190.5940) Self-action Effects.

\section{http://dx.doi.org/10.1364/ao.XX.XXXXXX}

The liquid crystalline phases feature unique optical properties, stemming from their hybrid nature between solids and liquids [1]. Liquid crystals (LCs) behave in general like inhomogeneous anisotropic materials with a locally varying optical axis, with important repercussions in photonics [2-4]. Between the numerous LC phases, the most investigated one is the nematic LC (NLC), featuring the absence of any positional order on the long range. Due to their fluid-like nature, NLC molecules are highly mobile, thus they undergo strong reorientation when subject to external electric fields. In the optical domain, fielddriven reorientation induces a very strong and nonlocal Kerr effect [5], yielding self-phase modulation [6], self-focusing [7] and, eventually, formation of self-trapped beams [8].

Nonetheless, a drawback comes from the high mobility of NLC molecules. With respect to solids, the lower spatial correlation in molecular position implies more time-dependent disorder. Due to the relatively stronger intermolecular interaction, the optical scattering in NLC is much higher (approximately six orders of magnitude) than in isotropic fluids [1]. As a matter of fact, NLC are an ideal benchmark for the investigation of light propagation in the multiple scattering regime, including light diffusion
$[9,10]$, weak localization [11] and random lasing [12].

In this Letter we investigate the interplay between dynamic scattering and light self-trapping in NLC. We model the molecular fluctuations with a Langevin force in the reorientation equation, the latter being solved in time together with the beam evolution. Our model accounts both for self-steering and longitudinal nonlocality. In the linear regime we observe the growth in propagation of a speckle field. When the power is sufficient for self-trapping, Brownian oscillations are observed, the latter being strongly enhanced when the light undergoes self-steering via nonlinear changes in the walk-off angle.

Let us consider a planar cell filled with uniaxial NLC. Hereafter we will neglect thermal nonlinearity, negligible in undoped NLC sufficiently far away from the isotropic-to-nematic transition [13]. The thickness along $x$ is $L_{x}=100 \mu \mathrm{m}$, whereas on the plane $y z$ the cell is assumed to be infinitely extended along $y$ and of length $L_{z}=1 \mathrm{~mm}$ along the propagation direction $z$. The optical properties are uniquely defined by the spatial distribution of the optical axis $\hat{n}$, which corresponds to the average orientation of the molecules also known as the director, and by the two eigenvalues $\epsilon_{\perp}$ and $\epsilon_{\|}$of the dielectric tensor $\epsilon$. The anchoring conditions are such that, in the absence of any external stimulus, the optical axis lies on the plane $y z$, forming an angle $\theta_{0}$ with respect to $\hat{z}$. Hereafter we will consider only $y$-polarized input beam, corresponding to extraordinary wave in the NLC. In the linear regime (i.e., small input powers), the extraordinary wave propagates in the medium subject to a spatial walk-off $\delta=\arctan \left(\frac{\epsilon_{y z}}{\epsilon_{z z}}\right)$, a refractive index $n_{e}=\sqrt{\left(\epsilon_{y y}-\frac{\epsilon_{y z}^{2}}{\epsilon_{z z}}\right) / \epsilon_{0}}$ and a non-unitary diffraction coefficient along $y, D_{y}=\frac{n_{e}^{2}}{\epsilon_{z z}}$ [14]. The optical torque is proportional to the optical anisotropy $\epsilon_{a}=\epsilon_{\|}-\epsilon_{\perp}$ and rotates the NLC molecules only in the plane $y z$. Thus, the angle $\theta=\angle(\hat{n} \hat{k})$ completely describes the system. The effects of the optical torque are accounted for by introducing $\psi=\theta-\theta_{0}$, where $\psi$ is the optical perturbation. In the nonlinear case, two different regimes are observed according to the ratio $r=\frac{\psi}{\theta}$. In the perturbative case $r$ is small, and the optically-induced rotation yields only a self-focusing effect, eventually generating spatial 
solitons. In the non-perturbative case $r$ is no more negligibly small: a self-steering effect due to the changes in the walk-off angle arises [14], the latter being due to the power-dependent changes in $\theta_{b}(z)$, where $\theta_{b}=\max (\theta)$ for a fixed $z$. Using a (1+1)D model, the magnetic field $H_{x}=A e^{i k_{0} n_{e}\left(\theta_{b}\right) z}$ propagates under the action of a photonic potential $\Delta n_{e}^{2}=n_{e}^{2}(\theta)-n_{e}^{2}\left(\theta_{b}\right)$ and a walk-off angle $\delta\left(\theta_{b}\right)=\delta_{b}$ according to [14, 15]

$$
\begin{aligned}
2 i k_{0} n_{e}\left(\theta_{b}\right)\left(\frac{\partial A}{\partial z}+\tan \delta_{b} \frac{\partial A}{\partial y}\right)+D_{y} \frac{\partial^{2} A}{\partial y^{2}}+k_{0}^{2} \Delta n_{e}^{2}(\theta) A=0 . \\
\begin{aligned}
\nu \frac{\partial \psi}{\partial t} & =K \nabla_{y z}^{2} \psi-\left(\frac{\pi}{L_{x}}\right)^{2} \psi \\
& +\frac{\epsilon_{0} \epsilon_{a}}{4} \sin \left[2\left(\theta_{0}+\psi-\delta_{b}\right)\right]\left|E_{t}\right|^{2}+\eta(y, z, t),
\end{aligned}
\end{aligned}
$$

where $k_{0}, v$, and $K$ are the vacuum wave number, the NLC viscosity, and the single elastic constant, respectively. Hereafter we will use physical parameters corresponding to the NLC E7. The transverse component $\boldsymbol{E}_{t}=-\frac{Z_{0}}{n_{e}\left(\theta_{b}\right) \cos \left(\delta_{b}\right)} H_{x} \hat{t}$ is the principal component of the electric field, directed along $\hat{t}=\cos \left(\delta_{b}\right) \hat{y}-\sin \left(\delta_{b}\right) \hat{z}$. Equation (1) premises a quasi-stationary regime for the light, that is, the optical field varies on much shorter time scales than the macroscopic perturbation $\psi$ governed by Eq. (2). As in the standard Langevin equation, the term $\eta(y, z, t)$ is the spatio-temporal thermal noise accounting for the fast microscopic motion. The stochastic term $\eta$ is a white Gaussian stochastic process both in space and in time, that is, $\left\langle\eta(\boldsymbol{r}, t) \eta\left(\boldsymbol{r}^{\prime}, t^{\prime}\right)\right\rangle=\eta_{0} \delta\left(\boldsymbol{r}-\boldsymbol{r}^{\prime}, t-t^{\prime}\right)$.

Following the approach described in the De Gennes's book
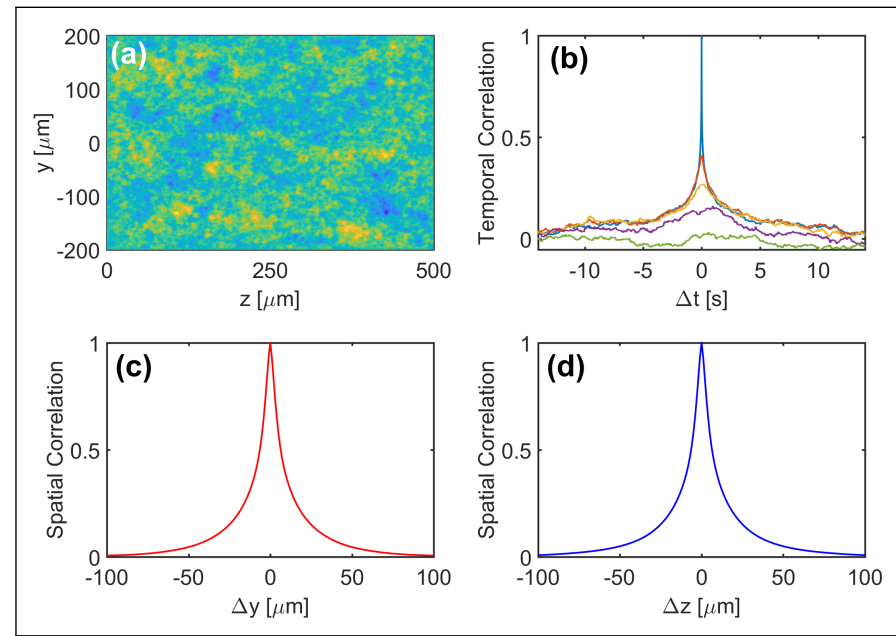

Fig. 1. Thermal fluctuations in a $200 \mu \mathrm{m} \times 400 \mu \mathrm{m}$ planar cell without illumination. (a) A typical realization for $\psi$. (b) Temporal correlation between the cell center and points distant $0,5,10,20,50 \mu \mathrm{m}$ along $y$, from top to bottom. (c-d) Spatial correlations along (c) $y$ and (d) $z$. The correlations are computed from a realization lasting $600 \mathrm{~s}$.

[1], in Ref. [16] the influence of scattering on light propagation has been accounted for by using the free energy of NLC plus the equipartition theorem. Here a different approach is used. We directly add the stochastic force $\eta$ to Eq. (2) [15, 17]: due to the strong intermolecular forces, the noise becomes colored, both in space and time. In this way, the longitudinal and transverse correlations in the noise pattern are properly accounted for. Our model does not account for scattering from extraordinary to ordinary and wide angle scattering (both small due to the momentum conservation), like back-scattering [18]. These two contributions act like an effective loss in the NLC $[14,16]$.

The first step is to investigate the thermal fluctuations without optical field, i.e., for $\boldsymbol{E}_{t}=0$. A snapshot is shown in Fig. 1(a), for a cell of sizes 200 and $400 \mu \mathrm{m}$ along $y$ and $z$, respectively. The average size of equally-orientated domains is dictated by the nonlocal response of the material [15]. An important point is to set the numerical parameters for an accurate modelling of the macroscopic fluctuations in $\psi$. For this purpose, we compute the temporal and spatial correlation from a solution of Eq. (2) of duration $600 \mathrm{~s}$. Figure 1(b) shows the temporal correlation between the cell center and four spatial points. The temporal correlation decreases with the reciprocal distance. With respect to the temporal delay $\Delta t$, the behavior at very short distances is fixed by the temporal step used in the numerical simulations due to the vanishing response time for local perturbations. Despite this, for longer distances the temporal correlation does not depend on the numerical step. In the presence of a random potential, the optical propagation is mainly affected by the spatially extended perturbations [16]. Finally, the spatial correlation is isotropic [Fig. 1(c-d)], with an average size of $20 \mu \mathrm{m}$ for each grain.

We now consider the propagation of low-power light beams

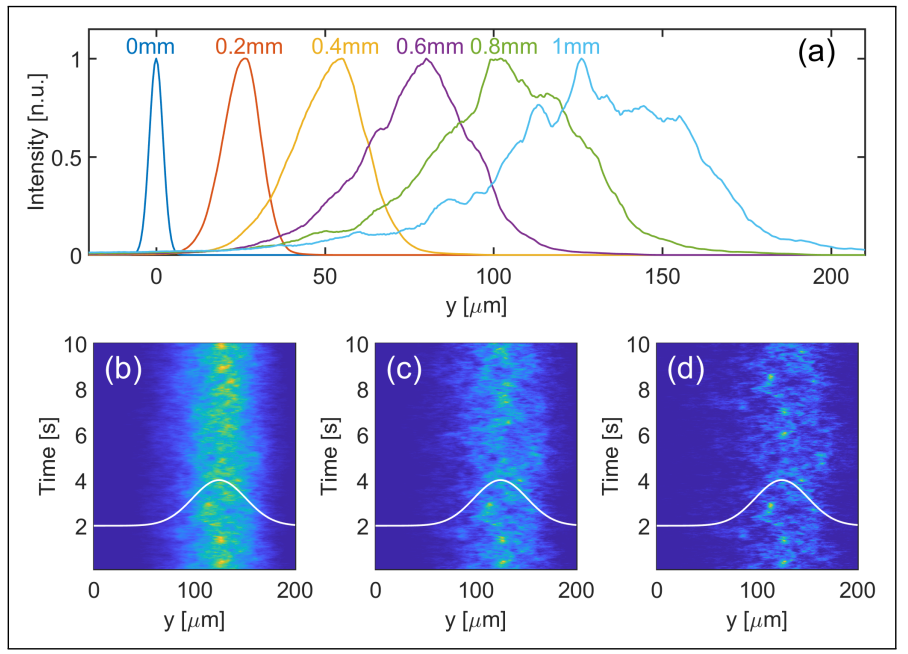

Fig. 2. Propagation of a Gaussian beam of waist $4 \mu \mathrm{m}$ in the linear regime. (a) Normalized average intensity versus $y$ at different distances $z$. Average is performed over $10 \mathrm{~s}$. (b-d) Instantaneous intensity distribution calculated in $z=1 \mathrm{~mm}$ versus the transverse coordinate $y$ for (b) $\sigma_{\psi}=0.16^{\circ}$, (c) $0.32^{\circ}$ and $(d) 0.63^{\circ}$. The white solid line is the intensity for $\eta_{0}=0$.

(i.e., negligible reorientational nonlinearity) in the presence of director fluctuations. Hereafter, a wavelength of $1064 \mathrm{~nm}$ will be considered. Equation (2) is solved using a first-order RungeKutta method with a temporal step of $1 \mathrm{~ms}$. The Langevin term $\eta$ is updated at each temporal step. The reorientational equation is solved on a numerical grid with transverse and longitudinal steps equal to $\Delta y=0.5 \mu \mathrm{m}$ and $\Delta z=2 \mu \mathrm{m}$, respectively (the longitudinal step used to solve Eq. (1) is 20 times smaller). The solution is saved every $10 \mathrm{~ms}$. The initial coherence of the beam monotonically fades out as light propagates inside the NLC [19], see Fig. 2(a) where the averaged intensity $\bar{I}=\frac{Z_{0}}{2 n_{e}^{2} \cos \delta T} \int_{0}^{T}|A(y, z, t)|^{2} d t$ is plotted. Unlike the quasiGaussian profile for $\eta_{0}=0, \bar{I}$ develops exponential tails due to 
the scattered light. For long enough distances, $\bar{I}$ tends to $e^{-|x| / L}$, i.e., the diffusive regime is achieved [9]. Figure 2(b-d) and Visualization 1 shows the temporal evolution versus the noise strength $\eta_{0}$, expressed through the physically relevant quantity $\sigma_{\psi}\left(\eta_{0}\right)=\sqrt{\left\langle(\psi-\langle\psi\rangle)^{2}\right\rangle}$, where the brackets correspond to a temporal average. For a fixed propagation distance larger fluctuations (i.e., bigger $\sigma_{\psi}$ ) lead to the formation of a speckle-like field with a lower amount of coherence.

Next we consider the light propagation in the presence of a sub-

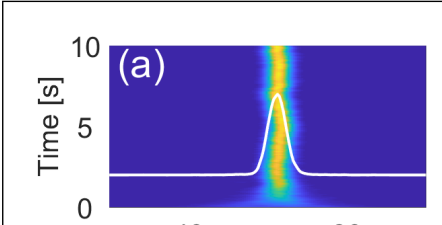

40

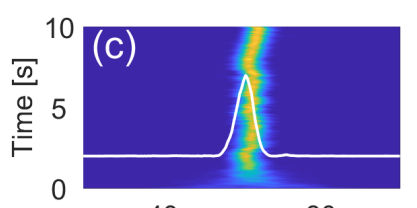

40

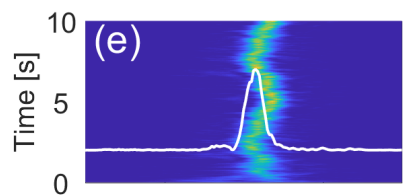

40

80

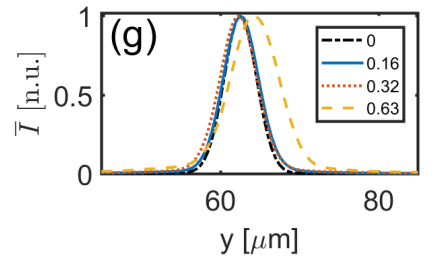

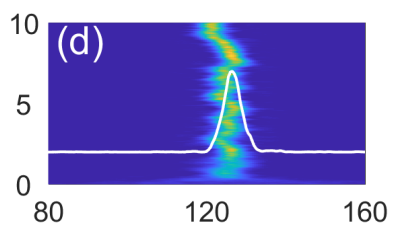
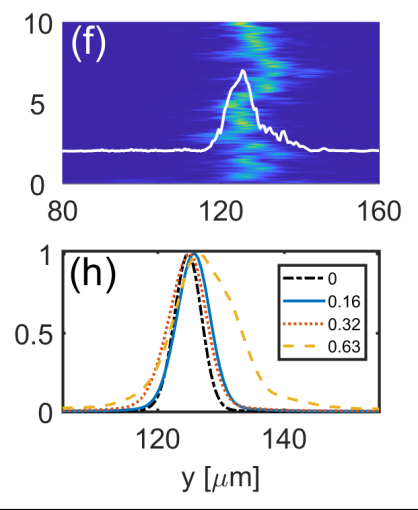

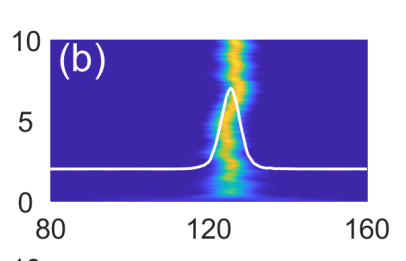

Fig. 3. Stable solitonic regime: propagation of a Gaussian beam of waist $4 \mu \mathrm{m}$ for $P=0.5 \mathrm{~mW}$, turned on at $t=0 \mathrm{~s}$. Intensity cross-sections versus $y$ and time in $(\mathrm{a}, \mathrm{c}, \mathrm{e}) z_{\mathrm{s}}=0.5 \mathrm{~mm}$ (mid-plane) and in (b,d,f) $z_{s}=1 \mathrm{~mm}$ (output facet); solid white lines correspond to the snapshot at $t=2 \mathrm{~s}$. Fluctuation levels are (a-b) $\sigma_{\psi}=0.16^{\circ}$, (c-d) $0.32^{\circ}$ and (e-f) $0.63^{\circ}$. Average intensity over $10 \mathrm{~s}$ at $(\mathrm{g}) z=0.5 \mathrm{~mm}$ and $(\mathrm{h}) z=1 \mathrm{~mm}$.

stantial amount of reorientational nonlinearity. A self-trapped beam is formed despite the fluctuations [20, 21]. Figure 3(a-f) and Visualization 2 report the temporal evolution of the crosssection of $\bar{I}$ at the cell mid-plane and at the output facet, for three different values of $\sigma_{\psi}$. The highly nonlocal response minimizes the losses due to the scattering events [15] (in real samples, losses are due to wide-angle scattering and extraordinary-to-ordinary conversion [18], both neglected here). The localized wave wiggles around the unperturbed $\left(\eta_{0}=0\right)$ solution, with the flickering motion increasing with $\eta_{0}$. The random inhomogeneities result in diffusive exponential tails in the cross-section of $\bar{I}$, see Fig. 3(g-h). The amplitude of the oscillations increase with the propagation distance $z$ due to the Brownian motion [15, 22]. At the same time, for larger $\sigma_{\psi}$ the diffusion becomes strong enough to induce substantial variations into the cross-section of $\bar{I}$.

Light behavior qualitatively changes when the power is large enough to induce self-steering via nonlinear changes in the walk-
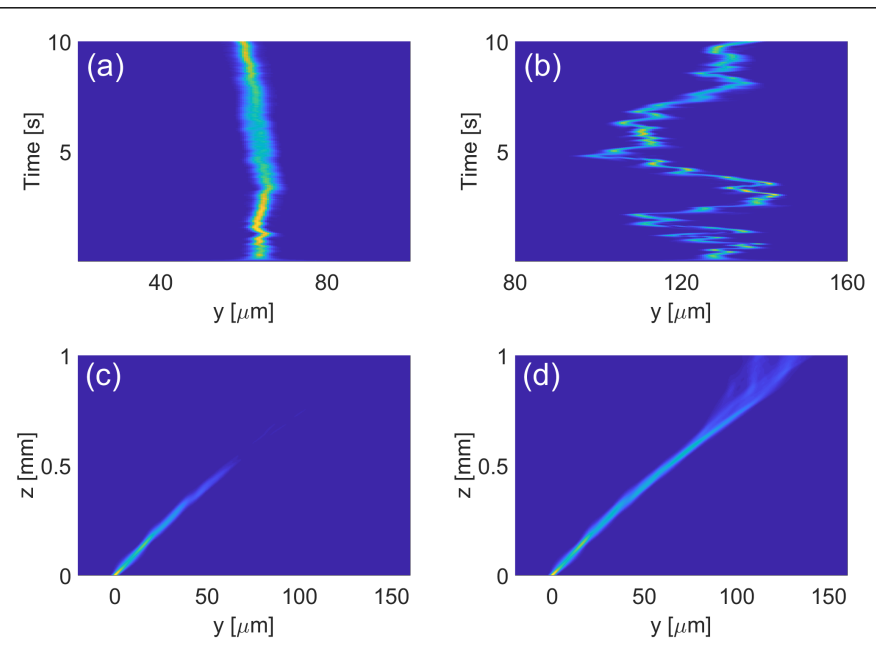

Fig. 4. Unstable solitonic regime: propagation of a Gaussian beam of waist $2 \mu \mathrm{m}$ for $P=2 \mathrm{~mW}$, turned on at $t=0 \mathrm{~s}$. Intensity cross-sections versus $y$ and time in (a) $z_{s}=0.5 \mathrm{~mm}$ (cell mid-plane) and in (b) $z_{\mathcal{S}}=1 \mathrm{~mm}$ (output facet). Plots on the plane $y z$ of (c) the square modulus of the averaged field and of (d) the average of the intensity. Fluctuation level is $\sigma_{\psi}=0.16^{\circ}$, whereas temporal averages are computed over $10 \mathrm{~s}$.

off angle [14]. Figure 4 shows the results for $P=2 \mathrm{~mW}$. For $\sigma_{\psi}=0.16^{\circ}$ the maximum shift reaches $\approx 20 \mu \mathrm{m}$. Looking at the temporal dynamics [Fig. 4(a-b) and Visualization 3], a large oscillation on the temporal scale of few seconds is superposing to the Brownian flickering. In Fig. 4(c-d) the square modulus of the averaged field $\bar{A}=\frac{1}{T} \int_{0}^{T} A(y, z, t) d t$ and the averaged intensity $\bar{I}$ are plotted, respectively. The quantity $\bar{A}$ estimates the temporal coherence by accounting for the phase [15]. The temporal coherence decays with $z$, achieving a fully incoherent field in $z \approx 500 \mu \mathrm{m}$. On the other hand, $\bar{I}$ is strictly related to the probability distribution of the field intensity. The temporal oscillations result in the broadening of the distribution along $y$, the lateral spread increasing with $z$. Physically, the Brownian motion shifts the beam away from the center of the self-induced waveguide. In turn, the beam is pushed back towards the equilibrium position (i.e., the solution for $\eta_{0}=0$ ) by a restoring force, the latter including contributions both from the index gradient and from the nonlinear changes in the walk-off [23]. The beam profile instantaneously responds to the perturbed index well, yielding strong changes in the light-written index distribution. The beam thus starts to oscillate around the equilibrium position. Summarizing, thanks to the nonlinear feedback, the microscopic motion induces a macroscopic motion of the whole beam.

Let us define the beam trajectory as the center of mass $y_{s}(z, t)=$ $\int y|A|^{2} d y / \int|A|^{2} d y$. Fig. 5(a) shows the standard deviation $\sigma_{p o s}=\sqrt{\left\langle\left(y_{s}(t)-\left\langle y_{s}\right\rangle\right)^{2}\right\rangle}$ for stable solitons. The trajectory spreading increases with $z$, with a trend more complicated than in Ref. [22] due to the longitudinal nonlocality. The mean path $\left\langle y_{s}\right\rangle$, plotted in Fig. 5(b), corresponds to the equilibrium position for $\eta_{0}=0$. In the unstable case [Fig. 5(c)], $\sigma_{\text {pos }}$ is still increasing with $z$, but the temporal average $\left\langle y_{s}\right\rangle$ differs from the equilibrium solution. Nonlocal longitudinal effects provide a strong feedback mechanism between different planes $z=$ const causing long-range fluctuations, see Fig. 5(d). 

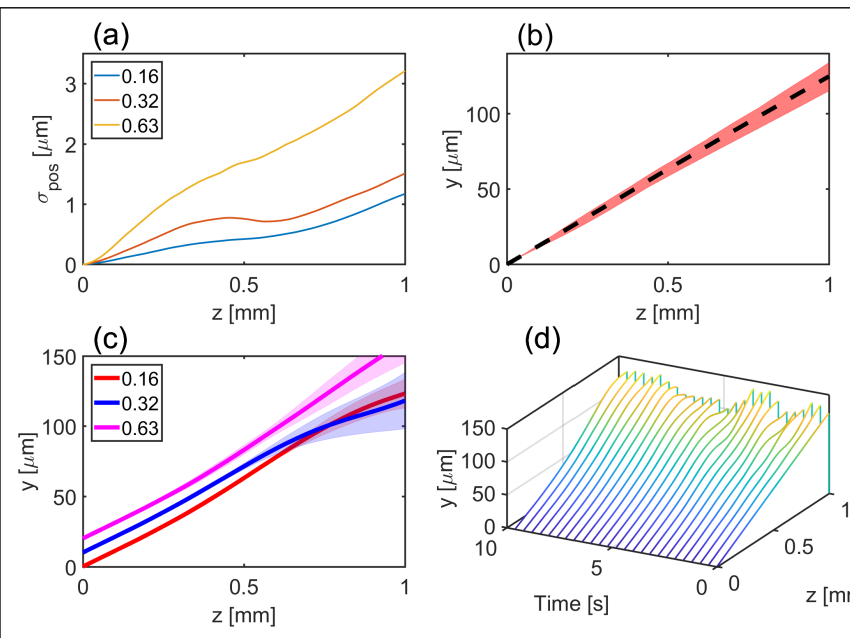

(d)

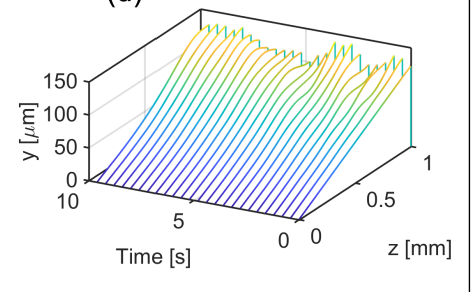

Fig. 5. (a) Standard deviation of $y_{S}$ versus $z$ when $P=0.5 \mathrm{~mW}$, for three values of $\sigma_{\psi}$. (b) Mean trajectory (dashed line) and realizations over $10 \mathrm{~s}$ (spanning the red area) when $\sigma_{\psi}=0.63$. (c) Beam position versus $z$ for $P=2 \mathrm{~mW}$ for different $\sigma_{\psi}$; the curves are vertically shifted ( $\sigma_{\psi}$ increases from bottom to top) to improve the visibility. Solid lines are the average, and the realizations span the shaded areas. (d) Set of trajectories versus time for $\sigma_{\psi}=0.16$. The averaging time is $10 \mathrm{~s}$.

Experimentally, we coupled an optical fiber with a core diameter of $2.9 \mu \mathrm{m}$ excited at $\lambda=1064 \mathrm{~nm}$ into a planar cell of thickness $75 \mu \mathrm{m}$, filled up with the NLC E7 [16]. The efficiency for the laser-to-fiber coupling is about $40 \%$ (here we report the power emitted by the laser). The comparison with the simulations must account for the fact that the theoretical power is $3 \sim 5$ times lower than actual powers [14]. Fig. 6 summarizes the behavior in the presence of self-confinement. As predicted, the beam wiggles in time, regardless of the power. The standard deviation $\sigma_{\text {pos }}$ increases with $z$, the trend being in good agreement with the simulations (see Fig. 5 and Visualization 2-5). The larger the input power the stronger the temporal fluctuations are: a significant increase is observed when self-steering starts to occur (see the behavior for $P=9.2 \mathrm{~mW}$ ).

In conclusion, we discussed the interplay between multiple scattering and nonlinear effects in NLCs. We showed that thermal fluctuations in the molecular director induce a permanent flickering of the self-localized beam. In the presence of self-steering, the self-focused beam is temporally unstable and oscillates with amplitudes of several beam widths, as originally reported by Braun in capillaries $[7,24]$. Our results are a starting point towards the improvement of the coherence and of the temporal stability of spatial solitons in liquid crystals, two detrimental effects hindering practical applications of these waves.

Funding. Deutsche Forschungsgemeinschaft (DFG) (GRK 2101); Belgian Science Policy Office (BELSPO) (IAP7-35); Fund for Research Training in Industry and Agriculture (FRIA); Fonds David et Alice Van Buuren; Fondation Jaumotte-Demoulin.

\section{REFERENCES}

1. P. G. DeGennes and J. Prost, The Physics of Liquid Crystals (Oxford Science, New York, 1993).

2. M. Čančula, M. Ravnik, I. Muševič, and S. Žumer, Opt. Express 24, 22177 (2016).

3. J. Kobashi, H. Yoshida, and M. Ozaki, Nat. Photon. 10, 389 (2016).
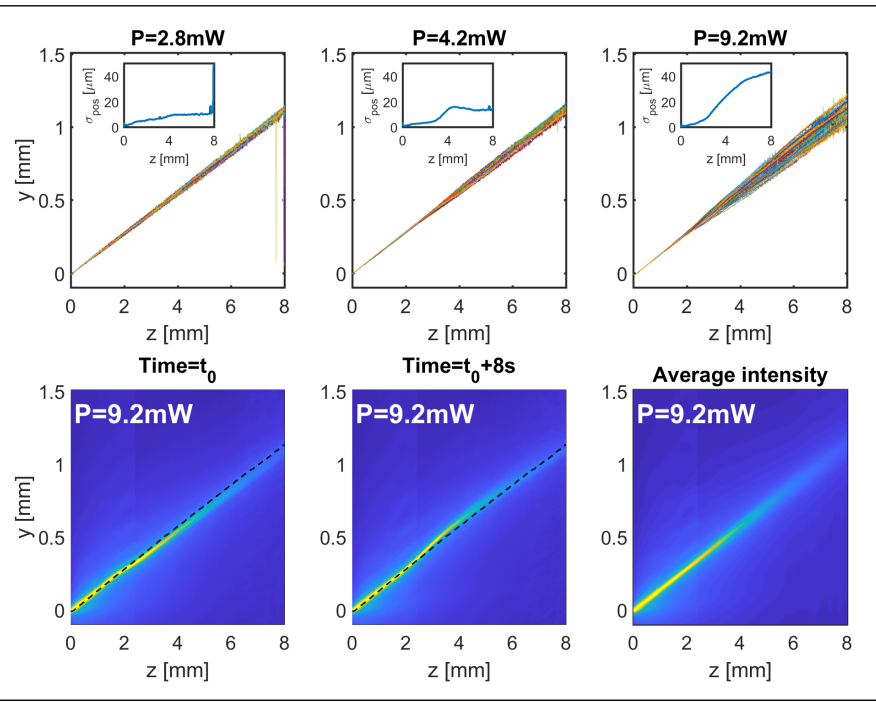

Fig. 6. Experimental results. Top row: collection of 101 beam trajectories for fixed input powers. Inset: the corresponding $\sigma_{\text {pos }}$ versus $z$. Bottom row: scattered light distribution on the plane $y z$ for $P=9.2 \mathrm{~mW}$. The first two columns are snapshots with a temporal lag of $8 \mathrm{~s}$ (black dashed line is the trajectory of the average field), whereas the latter column is the corresponding average intensity. The overall temporal window is $10 \mathrm{~s}$, whereas the acquisition time for a single snapshot is $60 \mathrm{~ms}$.

4. R. Barboza, U. Bortolozzo, S. Residori, and M. G. Clerc, Phys. Rev. Lett. 117, 053903 (2016).

5. N. Tabiryan and B. Zeldovich, Mol. Cryst. Liq. Cryst. 62, 237 (1980).

6. S. D. Durbin, S. M. Arakelian, and Y. R. Shen, Opt. Lett. 6, 411 (1981).

7. E. Braun, L. P. Faucheux, and A. Libchaber, Phys. Rev. A 48, 611 (1993).

8. M. Peccianti and G. Assanto, Phys. Rep. 516, 147 (2012).

9. B. A. van Tiggelen, R. Maynard, and A. Heiderich, Phys. Rev. Lett. 77, 639 (1996).

10. M. H. Kao, K. A. Jester, A. G. Yodh, and P. J. Collings, Phys. Rev. Lett. 77, 2233 (1996).

11. H. K. M. Vithana, L. Asfaw, and D. L. Johnson, Phys. Rev. Lett. 70, 3561 (1993).

12. G. Strangi, S. Ferjani, V. Barna, A. D. Luca, C. Versace, N. Scaramuzza, and R. Bartolino, Opt. Express 14, 7737 (2006).

13. F. Simoni, Nonlinear Optical Properties of Liquid Crystals (World Scientific, Singapore, 1997).

14. A. Alberucci, A. Piccardi, M. Peccianti, M. Kaczmarek, and G. Assanto, Phys. Rev. A 82, 023806 (2010).

15. F. Maucher, W. Krolikowski, and S. Skupin, Phys. Rev. A 85, 063803 (2012).

16. S. Bolis, S.-P. Gorza, S. J. Elston, K. Neyts, P. Kockaert, and J. Beeckman, Phys. Rev. A 96, 031803 (2017).

17. H. Louis, M. Tlidi, and E. Louvergneaux, Opt. Express 24, 16206 (2016).

18. A. Valkov and V. Romanov, JETP Lett. 63, 736 (1986).

19. A. Valkov, L. Zulkov, A. Kovshik, and V. Romanov, JETP Lett. 40, 1064 (1985).

20. Y. Lahini, A. Avidan, F. Pozzi, M. Sorel, R. Morandotti, D. N. Christodoulides, and Y. Silberberg, Phys. Rev. Lett. 100, 013906 (2008).

21. C. Conti, Phys. Rev. A 86, 061801(R) (2012).

22. V. Folli and C. Conti, Phys. Rev. Lett. 104, 193901 (2010).

23. A. Alberucci, A. Piccardi, U. Bortolozzo, S. Residori, and G. Assanto, Opt. Lett. 35, 390 (2010).

24. D. W. McLaughlin, D. J. Muraki, and M. J. Shelley, Physica D: Nonlinear Phenomena 97, 471 (1996). 


\section{FULL REFERENCES}

1. P. G. DeGennes and J. Prost, The Physics of Liquid Crystals (Oxford Science, New York, 1993).

2. M. Čančula, M. Ravnik, I. Muševič, and S. Žumer, "Anisotropic optical materials; liquid crystals; birefringence; polarization; self-focusing," Opt. Express 24, 22177-22188 (2016).

3. J. Kobashi, H. Yoshida, and M. Ozaki, "Planar optics with patterned chiral liquid crystals," Nat. Photon. 10, 389-392 (2016).

4. R. Barboza, U. Bortolozzo, S. Residori, and M. G. Clerc, "Berry phase of light bragg-reflected by chiral liquid crystal media," Phys. Rev. Lett. 117, 053903 (2016).

5. N. Tabiryan and B. Zeldovich, "The orientational optical nonlinearity of liquid-crystals," Mol. Cryst. Liq. Cryst. 62, 237-250 (1980).

6. S. D. Durbin, S. M. Arakelian, and Y. R. Shen, "Laser-induced diffraction rings from a nematic-liquid-crystal film," Opt. Lett. 6, 411-413 (1981).

7. E. Braun, L. P. Faucheux, and A. Libchaber, "Strong self-focusing in nematic liquid crystals," Phys. Rev. A 48, 611-622 (1993).

8. M. Peccianti and G. Assanto, "Nematicons," Phys. Rep. 516, 147 - 208 (2012).

9. B. A. van Tiggelen, R. Maynard, and A. Heiderich, "Anisotropic light diffusion in oriented nematic liquid crystals," Phys. Rev. Lett. 77, 639642 (1996).

10. M. H. Kao, K. A. Jester, A. G. Yodh, and P. J. Collings, "Observation of light diffusion and correlation transport in nematic liquid crystals," Phys. Rev. Lett. 77, 2233-2236 (1996).

11. H. K. M. Vithana, L. Asfaw, and D. L. Johnson, "Coherent backscattering of light in a nematic liquid crystal," Phys. Rev. Lett. 70, 3561-3564 (1993).

12. G. Strangi, S. Ferjani, V. Barna, A. D. Luca, C. Versace, N. Scaramuzza, and R. Bartolino, "Random lasing and weak localization of light in dyedoped nematic liquid crystals," Opt. Express 14, 7737-7744 (2006).

13. F. Simoni, Nonlinear Optical Properties of Liquid Crystals (World Scientific, Singapore, 1997).

14. A. Alberucci, A. Piccardi, M. Peccianti, M. Kaczmarek, and G. Assanto, "Propagation of spatial optical solitons in a dielectric with adjustable nonlinearity," Phys. Rev. A 82, 023806 (2010).

15. F. Maucher, W. Krolikowski, and S. Skupin, "Stability of solitary waves in random nonlocal nonlinear media," Phys. Rev. A 85, 063803 (2012).

16. S. Bolis, S.-P. Gorza, S. J. Elston, K. Neyts, P. Kockaert, and J. Beeckman, "Spatial fluctuations of optical solitons due to long-range correlated dielectric perturbations in liquid crystals," Phys. Rev. A 96, 031803 (2017).

17. H. Louis, M. Tlidi, and E. Louvergneaux, "Experimental evidence of dynamical propagation for solitary waves in ultra slow stochastic nonlocal kerr medium," Opt. Express 24, 16206-16211 (2016).

18. A. Valkov and V. Romanov, "Characteristics of propagation and scattering of light in nematic liquid crystals," JETP Lett. 63, 736-743 (1986).

19. A. Valkov, L. Zulkov, A. Kovshik, and V. Romanov, "Selective scattering of polarized light by an oriented liquid crystal," JETP Lett. 40, 10641067 (1985).

20. Y. Lahini, A. Avidan, F. Pozzi, M. Sorel, R. Morandotti, D. N. Christodoulides, and Y. Silberberg, "Anderson localization and nonlinearity in one-dimensional disordered photonic lattices," Phys. Rev. Lett. 100, 013906 (2008).

21. C. Conti, "Solitonization of anderson localization," Phys. Rev. A 86, 061801(R) (2012).

22. V. Folli and C. Conti, "Frustrated brownian motion of nonlocal solitary waves," Phys. Rev. Lett. 104, 193901 (2010).

23. A. Alberucci, A. Piccardi, U. Bortolozzo, S. Residori, and G. Assanto, "Nematicon all-optical control in liquid crystal light valves," Opt. Lett. 35, 390-392 (2010).

24. D. W. McLaughlin, D. J. Muraki, and M. J. Shelley, "Self-focussed optical structures in a nematic liquid crystal," Physica D: Nonlinear Phenomena 97, 471 - 497 (1996). 